\title{
Design and Performance of a DNP Prepolarizer Coupled to a Rodent MRI Scanner
}

\author{
A. COMMENT, ${ }^{1}$ B. VAN DEN BRANDT, ${ }^{2}$ K. UFFMANN, ${ }^{3}$ F. KURDZESAU, ${ }^{1,2}$ \\ S. JANNIN, ${ }^{1}$ J.A. KONTER, ${ }^{2}$ P. HAUTLE, ${ }^{2}$ W.TH. WENCKEBACH, ${ }^{1}$ \\ R. GRUETTER, ${ }^{3}$ J.J. VAN DER KLINK ${ }^{1}$ \\ ${ }^{1}$ Institut de Physique des Nanostructures (IPN), EPFL Station 3, CH-1015 Lausanne, Switzerland \\ ${ }^{2}$ Paul Scherrer Institute (PSI), CH-5232 Villigen, Switzerland \\ ${ }^{3}$ Laboratory for Functional and Metabolic Imaging (LIFMET), EPFL Station 6, CH-1015 Lausanne, Switzerland
}

\begin{abstract}
For most of the last forty years, the techniques of Dynamic Nuclear Polarization (DNP) have been confined to particle-physics laboratories building polarized targets, but recently it has been shown that samples similar to a solid target can be transformed into room temperature liquid solutions while retaining a high nuclear polarization. This method of "hyperpolarization" is of interest in NMR/MRI/MRS. We describe a 3.35 T DNP/9.4 T MRI installation based on a continuous-flow cryostat, using a standard wide-bore low-field NMR magnet as prepolarizer magnet and a widely available radical as polarizing agent. The interfacing to a rodent scanner requires that the infusion of the polarized solution in the animal be remotely controlled, because of limited access inside the magnet bore. Physiological constraints on the infusion rate can be a serious source of polarization loss, and the discussion of efficiency is therefore limited to that of the prepolarizer itself, i.e., the spin temperatures obtained in the solid state. To put our results in context, we summarize data obtained in targets with different types of radicals, and provide a short review of the DNP mechanisms needed in their discussion. (c) 2007 Wiley Periodicals, Inc. Concepts Magn Reson Part B (Magn Reson Engineering) 31B: 255-269, 2007
\end{abstract}

KEY WORDS: dynamic nuclear polarization; hyperpolarization; enhanced ${ }^{13} \mathrm{C}$ NMR

\section{INTRODUCTION}

Dynamic Nuclear Polarization (DNP) is the enhancement of nuclear spin polarization in a solid by transfer of polarization from the electron spins of paramagnetic centers that are fixed with respect to the nuclei, distinct from the Overhauser effect and the CIDNP effect. The most widespread application of

Received 15 July 2007; revised 17 July 2007; accepted 17 July 2007

Correspondence to: J.J. van der Klink; E-mail: sdnpi@epfl.ch

Concepts in Magnetic Resonance Part B (Magnetic Resonance Engineering), Vol. 31B(4) 255-269 (2007)

Published online in Wiley InterScience (www.interscience.wiley. com). DOI 10.1002/cmr.b.20099

(c) 2007 Wiley Periodicals, Inc.
DNP so far has been in particle physics, where it is used to study the spin-dependent properties of nuclei by scattering a beam of particles from the polarized nuclei of interest inside a sample of bulk matter, a "polarized target." Most polarized target development has been devoted to the polarization of protons and deuterons. Sample material is chosen to maximize the density of nuclei of interest, and in that respect an important step, as far as this article is concerned, has been the development of targets consisting of frozen beads of alcohol/water mixtures around 1969, for a review see (1).

In a schematic view of DNP processes, where one distinguishes only between the "solid effect" and "thermal mixing" (see Section II), the polarization of low- $\gamma$ nuclei in frozen glassy solvents with radicals usually proceeds through thermal mixing. This means 
that at the end of the polarization step, all nuclei in the sample (except perhaps the protons) end up at the same spin temperature $T_{\mathrm{s}}$, and therefore the efficiency of the process at a given applied field $B_{0}$ and bath temperature $T$ can be characterized by the value of $T_{\mathrm{s}}$. For compactness, we will refer to e.g., ${ }^{13} \mathrm{C}$ spin temperatures as ${ }^{13} T_{\mathrm{s}}\left(B_{0}, T\right)$. Early work on butanol/water samples, at $B_{0}=2.5 \mathrm{~T}$ and $T=0.54 \mathrm{~K}$ resulted in a ${ }^{13} \mathrm{C}$ spin temperature ${ }^{13} T_{\mathrm{s}}(2.5 \mathrm{~T}$, $0.54 \mathrm{~K})=3 \mathrm{mK}(2)$.

In the early 1980's a group led by Rob Wind developed a method that combined, at room temperature, DNP and ${ }^{13} \mathrm{C}$ MAS-NMR, in a program that studied properties of coals (3). Later Griffin et al.(4) demonstrated similar experiments on "target-type" samples, consisting of frozen beads of a glass-forming solvent that contained, in addition to a radical, the molecule to be studied. They have provided a detailed analysis of such experiments in (5).

Some of this work has yielded remarkable results: in stationary samples of radical-doped polystyrene, Wind's group cooled the proton spins in a room temperature solid sample in a $1.4 \mathrm{~T}$ field to obtain a polarization of $1,200 \mathrm{ppm},{ }^{1} T_{\mathrm{s}}(1.4 \mathrm{~T}, 295 \mathrm{~K})=1.2$ $\mathrm{K}$. An example from Griffin's group is a MAS experiment with a sample of ${ }^{13} \mathrm{C}$-glycine in glycerol at $90 \mathrm{~K}$ in a $5 \mathrm{~T}$ field, yielding a DNP-created proton polarization estimated as $2,500 \mathrm{ppm}$, that was subsequently transmitted by cross polarization to the ${ }^{13} \mathrm{C}$, resulting in ${ }^{13} T_{\mathrm{s}}(5 \mathrm{~T}, 90 \mathrm{~K})=0.5 \mathrm{~K}$.

An ingenious application of DNP to liquid-state NMR has recently been developed by Wolber, Ardenkjaer-Larsen, Golman and colleagues (6, 7). They have shown that it is actually possible to transform a frozen-beads sample into a polarized liquid room temperature solution by dissolving it rapidly in superheated water. The sample transfer between DNP-polarization and NMR-observation magnets can be done simply by blowing the solution from one to the other through a thin plastic tube. As paramagnetic center they used a proprietary triarylmethyl (TAM) radical. For certain samples, the enhancement of the liquid-phase ${ }^{13} \mathrm{C}$ signal was more than a factor $10^{4}$ in a $9.4 \mathrm{~T}$ field.

The main goal of the work described in this article is to polarize ${ }^{13} \mathrm{C}$ in molecules of metabolic interest, and to deliver them in a reproducible manner to a living animal inside a rodent-type MRI scanner (a Varian/Magnex 9.4 T instrument, installed at the EPFL by the CIBM collaboration). We want to use an easily available radical as the paramagnetic center. Because optimization of the polarization in the solid state is important, our design is a trade-off between ease of operation, NMR sensitivity in the solid state, required microwave power and liquid He consumption. Similarly, we want optimal control over the concentration and the volume of solution that is finally infused in the animal. Since we work with a high-field narrow-bore animal scanner, the infusion process must be controlled remotely. The operation sequence is a mix of asynchronous operator intervention and computer-timed steps.

The article is organized as follows. The Section II summarizes the characteristics of the main DNP mechanisms, and gives some experimental criteria to distinguish between them. In Section III we describe the hardware parts of our prepolariser. Typical performance is shown in Section IV, with examples of solid-state ${ }^{13} \mathrm{C}$ polarization of several small molecules. A full in vitro experiment, including dissolution and blow-over into the MRI magnet is given as well. These results are discussed in the context of earlier target experiments in Section V, where also the differences with the results using TAM radicals are pointed out.

\section{DNP MECHANISMS}

In many cases DNP can be understood considering two mechanisms, the "solid effect" and "thermal mixing." It should be stressed that these are really two different mechanisms and not two different descriptions of the same process. Though examples are known where both mechanisms operate simultaneously (8), in many cases DNP proceeds clearly either through the solid effect or through thermal mixing. Here we present a short description of both mechanisms aiming to provide some simple experimental criteria to distinguish between them; it does not pretend to be a complete theoretical survey, for which we refer to (9). Our samples, similar to target materials, are glasses and therefore show inhomogeneously broadened powder ESR spectra. A specific model for DNP in such cases is described in the "Borghini Model" section.

\section{Solid Effect}

Consider a spin pair consisting of an electron spin $S$ $=1 / 2$ and a nuclear spin $I=1 / 2$, in a strong magnetic field and at low temperature, typically $3.35 \mathrm{~T}$ and $1.2 \mathrm{~K}$ for our DNP experiments. Its thermal equilibrium is best visualized using Fig. 1, depicting vertically the energy and horizontally the diagonal components of the density matrix, which we denote by $\rho\left(m_{\mathrm{S}}, m_{\mathrm{I}}\right)$ where $m_{\mathrm{S}}$ and $m_{\mathrm{I}}$ are the quantum numbers describing $S_{\mathrm{z}}$ and $I_{\mathrm{z}}$. Applying a microwave field at 


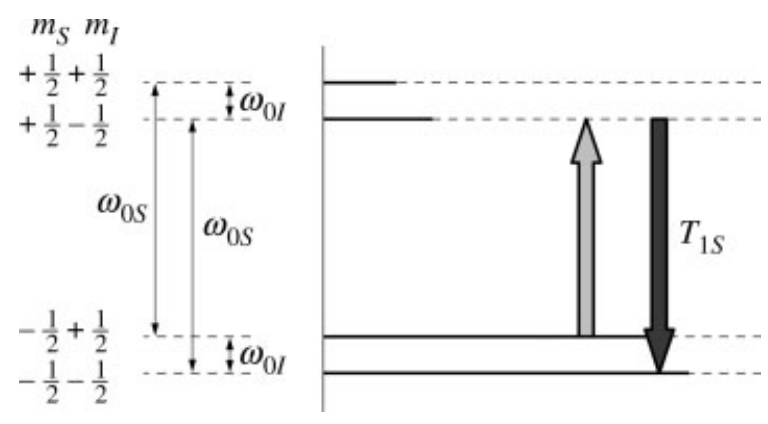

Figure 1 Diagram for the explanation of the solid effect. The left side sketches the energy level diagram for a pair of coupled spins-1/2. The right side sketches (very exaggerated) the thermal occupation of these levels. The light arrow indicates the pumping of a transition as described in the text, and the dark arrow the corresponding spinlattice relaxation.

the difference frequency $\left(\omega_{0 \mathrm{~S}}-\omega_{\mathrm{OI}}\right)$ pumps the twoquantum transition indicated by the light gray arrow, thus increasing $\rho(+1 / 2,-1 / 2)$ and decreasing $\rho(-1 /$ $2,+1 / 2)$ and hence transferring positive electron spin polarization into positive nuclear spin polarization. Observe that this is a two-quantum transition, which has to be "aided" by the dipolar interaction between the two spins.

This process is able to align just a single nuclear spin. However, after electron spin-lattice relaxation $T_{1 \mathrm{~S}}$ has restored thermal equilibrium with the lattice through the transition depicted by the dark gray arrow, the process can be repeated for other nuclear spins. Of course the process has to compete with nuclear spin-lattice relaxation (not pictured), which acts as a leak of nuclear spin polarization. Pumping at the sum frequency $\left(\omega_{0 \mathrm{~S}}+\omega_{0 \mathrm{I}}\right)$ has the opposite effect and transfers positive electron spin polarization into negative nuclear spin polarization.

The solid effect is generally observed if the width of the ESR line is significantly narrower than the NMR frequency $\omega_{0 I}$ resulting in the typical result for the nuclear polarization as a function of the microwave frequency $\omega_{\mathrm{m}}$ shown in Fig. 2. Note especially the flat region around $\omega_{\mathrm{m}}=\omega_{0 \mathrm{~s}}$. This situation is called the well resolved solid effect. If the NMR frequency is comparable to the ESR line width, the two pumping processes compete; a situation known as the differential solid effect. This would show in Fig. 2 as a partial or whole overlap between the left hand part leading to positive nuclear spin polarization and the right hand part leading to negative nuclear spin polarization.

Apart from the typical dependence on the microwave frequency shown in Fig. 2, the well resolved solid effect is experimentally often characterized by the need of considerable microwave power (due to the fact that a "forbidden" flip-flip two-quantum transition is pumped) and by equal final nuclear spin polarizations rather than equal spin temperatures. The maximum obtainable polarization may depend on the concentration of electron spins, because of the competition between the pumping process and nuclear spin-lattice relaxation.

\section{Thermal Mixing}

Thermal mixing is a different path for DNP, which can be effective instead of, or parallel to the solid effect. Contrary to the solid effect where the nuclei are polarized in a single step by means of a twoquantum transition, DNP via thermal mixing is a two step process where the first step is a single quantum transition. Thus, thermal mixing generally requires less microwave power than the solid effect. For efficient thermal mixing generally the width of the ESR line must be comparable to or larger than the NMR frequency. The concentration of electron spins must be high enough, so that their dipolar coupling can establish an electron spin temperature. If these conditions are satisfied thermal mixing is almost always the experimentally dominant mechanism, because less microwave power is needed.

Dynamic Cooling. The "single quantum" first step is best described using a fundamental idea due to Redfield (10) in the context of NMR, and that has been applied to ESR and DNP in (11). When a radiofrequency or microwave field is applied to a spin system close to its Larmor frequency, the effective Zeeman Hamiltonian is time-independent in a frame rotating together with that field around the static field, and the large Zeeman part of the static-frame Hamiltonian is considerably reduced. The idea is that nevertheless thermodynamic considerations of entropy maximalisation apply in the rotating frame.

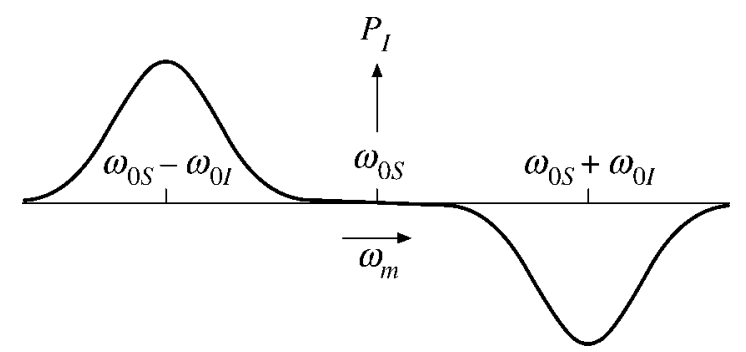

Figure 2 Typical result for the nuclear spin polarization $P_{\mathrm{I}}$ as a function of the pumping microwave frequency $\omega_{\mathrm{m}}$ under the well-resolved solid effect. In general, such a graph is known as a microwave spectrum. 


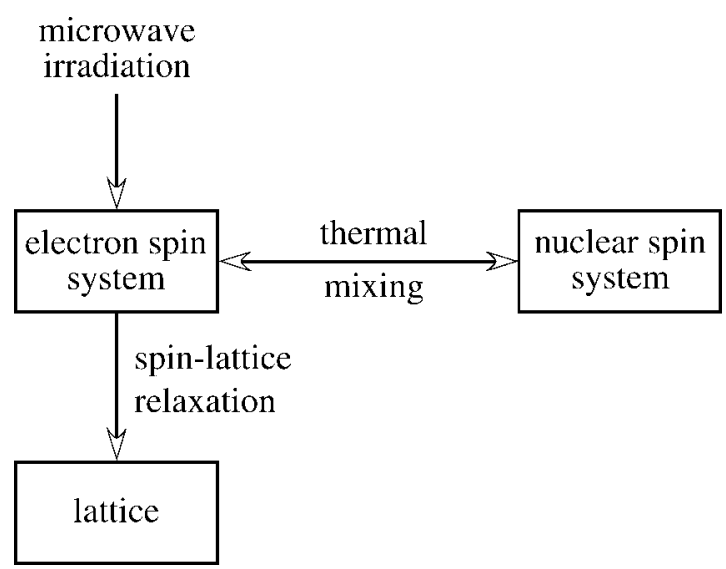

Figure 3 Schematic picture of the processes involved in DNP via thermal mixing.

That implies that the magnetization in this frame can be described by the Brillouin function with an appropriate "spin temperature in the rotating frame" $T_{\mathrm{r}}$ just as it can be characterized by the lattice temperature $T$ in the laboratory frame, when in thermal equilibrium with the lattice.

Consider irradiation of the sample with a microwave field at a frequency $\omega_{\mathrm{m}}$, which differs from the ESR Larmor frequency $\omega_{0 S}$. If the microwave field is strong enough, then according to eq. 38 of (10), in the rotating frame all electron spins acquire a single spin temperature $T_{\mathrm{r}}$ obeying

$$
T_{\mathrm{r}}=\frac{\Delta_{0}^{2}+a D^{2}}{\omega_{0 S} \Delta_{0}} T
$$

Here $T$ is the temperature of the lattice (in practice: of the cryostat bath) and $\Delta_{0}=\omega_{0 \mathrm{~S}}-\omega_{\mathrm{m}} . D^{2}$ is the dipolar second moment of the ESR line, and $a$ a model-dependent numerical constant of the order of $a \approx 2$. It is assumed that the squared offset-field is much larger than the square of the microwave field. The total process is pictured by the two vertical arrows in Fig. 3.

Clearly $\left|T_{\mathrm{r}}\right|$ can be orders of magnitude lower than $T$; this is the phenomenon called "dynamic cooling." Moreover, $T_{\mathrm{r}}$ follows the sign of the offset frequency $\Delta_{0}$.

Thermal Mixing. The second step to obtain DNP by this process is the "thermal mixing in the strict sense," whereby this low electron spin temperature in the rotating frame $T_{\mathrm{r}}$ is communicated to the nuclear spin temperature $T_{\mathrm{s}}$ in the laboratory frame. There are various ways to describe this step, but for our present purposes it is most convenient to describe it as a relaxation process involving a simultaneous transition of two electron spins $S^{1}$ and $S^{2}$ and one nuclear spin $I$ of the type $\delta m_{\mathrm{S} 1}= \pm 1, \delta m_{\mathrm{S} 2}=\mp 1$ and $\delta m_{\mathrm{I}}=\mp 1$. For such transitions to occur we need a mutual interaction between the electron spins (thus again requiring a not too low concentration) and a dipolar interaction between one of them and the nuclear spin. Furthermore, conservation of energy requires that the difference of ESR frequency of the two electron spins is equal to the NMR frequency. But for such pairs of electron spins to exist, the ESR line width needs to be of the order of the NMR frequency or broader, which is the requirement already mentioned earlier.

From thermodynamic considerations one has to conclude that this second step, the thermal mixing process proper, tends to equalize the spin temperature of the electron spins in the rotating frame $T_{\mathrm{r}}$ and the spin temperature of the nuclear spins in the laboratory frame of reference $T_{\mathrm{s}}$. As in molecular thermodynamics, the common final temperature will depend on the "heat capacity" of the two systems. Moreover, in the inverse direction the thermal mixing process generally provides the main path for nuclear spinlattice relaxation. The processes that are involved in this second step are represented by the horizontal double arrow and the lower vertical arrow in Fig. 3.

Thus, if several spin species are present, a typical feature of DNP via thermal mixing is that they acquire equal spin temperatures rather than equal polarizations. Furthermore, the maximum obtainable nuclear spin polarization depends on $T_{\mathrm{r}}$ only, so it is fundamentally independent of the concentration of electron spins. On the other hand one should be aware that the number of electron spins is generally much smaller than the number of nuclear spins, so the heat capacity of the electron spin system in the rotating frame is also much smaller than the heat capacity of the nuclear spin systems involved. Therefore, the achievable rate of dynamic cooling can be severely limited by the nuclear spins (and e.g., proton spins form a bigger "heat load" on the electron spin system than do the same number of carbon spins). To explain an experiment of DNP by thermal mixing in detail, the rate equations involving all processes need to be solved.

\section{Borghini Model}

This model (12) attempts to calculate the equivalent of eq. 1 for the case of an ESR powder spectrum with $g$-anisotropy. Once the result for $T_{\mathrm{r}}$ has been obtained, thermal mixing determines the effect on the nuclear spin temperature $T_{\mathrm{s}}$ along the lines of "Thermal Mixing" sub section. 
The ESR spectrum is a superposition of resonances from different radicals $i=1, \ldots, N$ with distinct orientations $\left(\theta_{i}, \phi_{i}\right)$ of the applied field $\mathbf{B}$ with respect to the principal axes of the $g$-tensor, and the effective value of $g$ is given by:

$$
\begin{aligned}
g_{i}^{2}= & g_{1}^{2} \cos ^{2}\left(\theta_{i}\right)+g_{2}^{2} \sin ^{2}\left(\theta_{i}\right) \cos ^{2}\left(\phi_{i}\right) \\
& +g_{3}^{2} \sin ^{2}\left(\theta_{i}\right) \sin ^{2}\left(\phi_{i}\right)
\end{aligned}
$$

For ease of discussion, the spectrum is considered to consist of $i=1, \ldots, N$ resonances, at relative frequency $\Delta_{i}$ from the center of the line, and with normalized intensities $f\left(\Delta_{i}\right)$ :

$$
\begin{gathered}
\sum_{i} f\left(\Delta_{i}\right)=1 \\
\sum_{i} \Delta_{i} f\left(\Delta_{i}\right)=0
\end{gathered}
$$

where the last equation defines the center of the line. The ESR frequency at this point is $\omega_{0 s}$. Now we switch on a microwave field at frequency $\omega_{\mathrm{m}}$, which corresponds to an offset $\Delta_{0}$ with respect to $\omega_{0 S}$. The hypothesis of the Borghini model is that electron dipole-dipole interactions, although weak enough to be unimportant for the line shape, will nevertheless maintain a unique spin temperature $T_{\mathrm{r}}$ among the resonances $i$. The role of the electron dipole-dipole interaction is thus reduced to establishing the temperature $T_{\mathrm{r}}$, but its contribution to the energy is neglected. Furthermore, it is assumed that the average of the squared offset-field is much larger than the square of the microwave field. With those approximations, the eq. 1 is replaced by the solution of

$$
\begin{gathered}
\tanh \left(\frac{\hbar \omega_{0 \mathrm{~S}}}{2 k T}\right)=\sum_{i} f\left(\Delta_{i}\right) \tanh \left(\frac{\hbar\left(\Delta_{0}-\Delta_{i}\right)}{2 k T_{\mathrm{r}}}\right) \frac{\Delta_{0}-\Delta_{i}}{\Delta_{0}} \\
\quad=\int_{-\infty}^{\infty} f(\Delta) \tanh \left(\frac{\hbar\left(\Delta_{0}-\Delta\right)}{2 k T_{\mathrm{r}}}\right) \frac{\Delta_{0}-\Delta}{\Delta_{0}} d \Delta
\end{gathered}
$$

where in the last line $f(\Delta)$ is the shape function of the ESR line. For a derivation of this result see e.g., eq. 6.102 in (9). Now a further hypothesis is that thermal mixing (in the strict sense) transmits this same temperature $T_{\mathrm{r}}$ to the nuclear spins in the laboratory frame, as described in "Thermal Mixing" section.

As a simple, although rather unrealistic, numerical example of the use of eq. 5, suppose that the experimental ESR line shape is Gaussian with second moment $D_{\mathrm{p}}^{2}$, where $D_{\mathrm{p}} \propto B_{0}$ (the index p stands for powder), and for ease of argument we call $D_{\mathrm{p}} / B_{0}=$

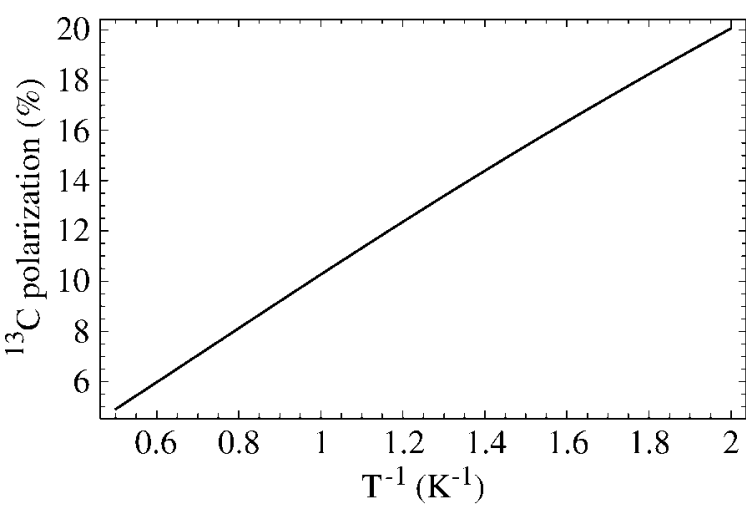

Figure 4 Optimal ${ }^{13} \mathrm{C}$ polarization in $3.35 \mathrm{~T}$ as function of inverse lattice temperature for a Gaussian ESR line. See text for line width parameter.

$\Delta g / g$, where $\Delta g$ is a measure for the spread in the $g_{1}$, $g_{2}, g_{3}$ values in eq. 2. For a given $\Delta g / g$ parameter, we can calculate the optimal ${ }^{13} \mathrm{C}$ polarization as a function of inverse lattice temperature $T^{-1}$ at constant field, and a result is shown in Fig. 4. Here we have chosen $\Delta g / g=4.65 \times 10^{-3}$, so that the ${ }^{13} \mathrm{C}$ polarization comes out as $8 \%$ at $1.2 \mathrm{~K}$ (compare Section IV). The nuclear polarization increases linearly with $T^{-1}$, although the electron polarization saturates, see Fig. 5. Similarly, the ${ }^{13} \mathrm{C}$ polarization is linear in the applied field, see Fig. 6.

These calculations can of course be done for more realistic line shapes, including the effects of spin-lattice relaxation (that are temperature and field dependent). Such very complete calculations have been performed in (8). The main goal of these illustrations was to show that, if indeed this model is applicable, the ${ }^{13} \mathrm{C}$ polarizations are expected to be linear in applied field and inverse bath temperature. This argument will be used in the construction of Table 1 in Section V.

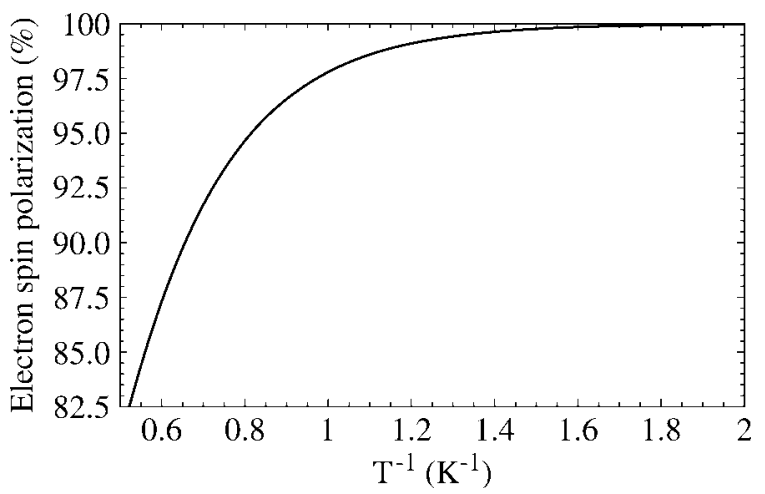

Figure 5 The electron polarization as function of the inverse lattice temperature in $3.35 \mathrm{~T}$. 


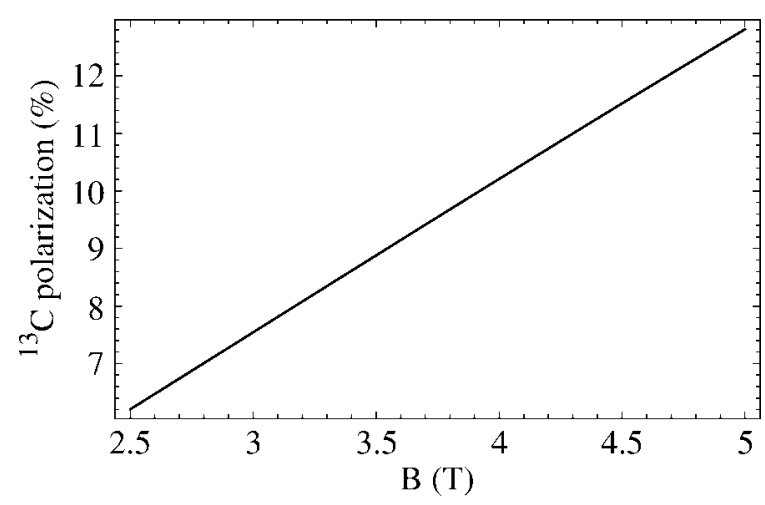

Figure 6 Optimal ${ }^{13} \mathrm{C}$ polarization at $1.2 \mathrm{~K}$ as function of magnetic field. The assumed ESR line width is the same as in Fig. 4.

\section{MAIN HARDWARE COMPONENTS}

The main components of our installation that we discuss in more detail in the following are

1. A continuous-flow ${ }^{4} \mathrm{He}$ cryostat, with associated pumping system.

2. The cryostat insert that contains the microwave cavity and the NMR coil. It also holds the connection between NMR coil and the top of the cryostat.

3. The sample holder insert, a simple straight tube with an exchangeable sample holder at its bottom. It serves to raise and lower the sample to its place inside the NMR coil and microwave cavity of the cryostat insert.

4. The waveguide insert. This is fitted to the main and sample holder inserts during microwave irradiation. Its main parts are a circular waveguide, and a simple coupling structure, to couple the guide to the microwave cavity.

5. The dissolution and blow-over insert. This is fitted to the cryostat and sample-holder inserts after the microwave irradiation. It contains on top the water boiler reservoir, with temperature and pressure transducers, and also the ingoing solvent and outgoing solution lines. The lower ends of these lines go into the sample holder.

6. The separator/infusion pump. This part is installed inside the imager magnet, close to the animal. Since the blow-over is performed using He gas, the gas must be separated from the solution before infusion.

Furthermore, the setup requires a polarizer magnet, a simple NMR spectrometer and a microwave source. The easy availability of microwave components at $94 \mathrm{GHz}$ makes this frequency (of interest in communication technologies) a typical choice for the ESR. It can be convenient to recycle a surplus lowfield NMR magnet as the polarizer magnet. The homogeneity required by the experiment can easily be obtained by just roughly adjusting the linear shims. It is of no great disadvantage if the magnet is not shielded: at the required field of $3.35 \mathrm{~T}$ the $5 \mathrm{mT}$ line will typically be less than $1 \mathrm{~m}$ outside the outer vacuum case, which translates into an extra second of blow-over time with respect to a shielded magnet.

The NMR spectrometer can be rather simple, but should have sufficient output power: because the lower parts of the sample holder and dissolution/blowover inserts actually pass inside the NMR coil, its filling factor is unfavorable, whereas, in particular for protons, the solid NMR line widths are rather large.

Suitable solid-state microwave sources are commercially available. We use a $400 \mathrm{~mW}$ unit (Elva-1, model SOM-10/94/400), with frequency stabilization by a PLL, but none of our experiments so far has required more than $30 \mathrm{~mW}$.

The preparation of the initial frozen beads is done manually, and those of poor quality (visual inspection turns out to be a reliable criterion) are discarded. For some experiments, we choose a small number of beads, with a high concentration of relevant molecules; for others solubility may require a larger frozen-sample volume. Although the filling factor for beads is less than for solid slugs, the better quality of the glass so formed improves homogeneity, and the sample temperature will be more uniform.

\section{Cryostat}

The design considerations for the cryostat, see Fig. 7, are set by the $88 \mathrm{~mm}$ room-temperature bore diameter of the polarizer magnet, and by the space in between the turrets on its top. For ease of operation, we have preferred to omit liquid nitrogen precooling, and use gas cooled shields instead. Two such shields are necessary for efficient operation. The wish for a sturdy design that avoids critically small clearances between the rather long shields, and the use of standard tubing sizes, brings the dimensions of the tail section to $56 \mathrm{~mm}$ inside diameter for the liquid He reservoir and $80 \mathrm{~mm}$ outside diameter for the outer mantle, with two copper heat shields in between. The diameter of the central access tube to the liquid $\mathrm{He}$ reservoir is $37 \mathrm{~mm}$. The neck of the cryostat, that sits on top of the magnet's outer vacuum case in between the turrets, has an outer diameter of $160 \mathrm{~mm}$. The top flange (diameter $225 \mathrm{~mm}$ ) is mounted to the outer 


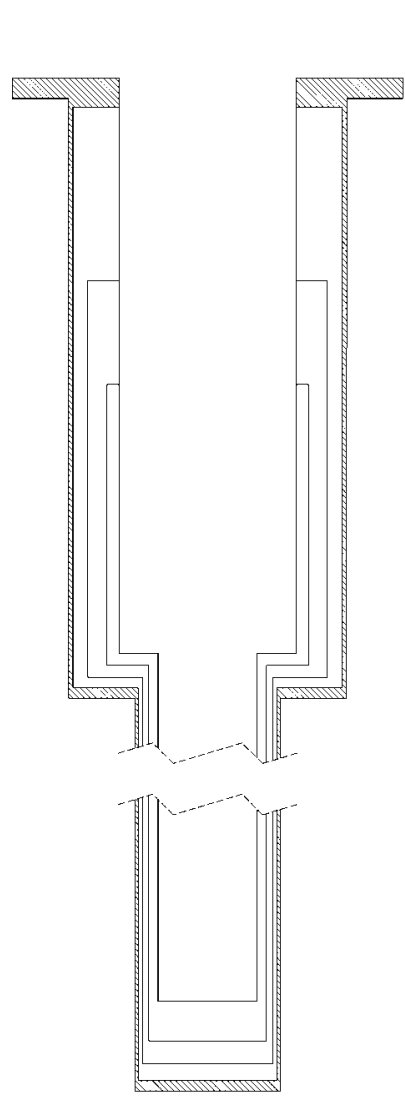

(a)
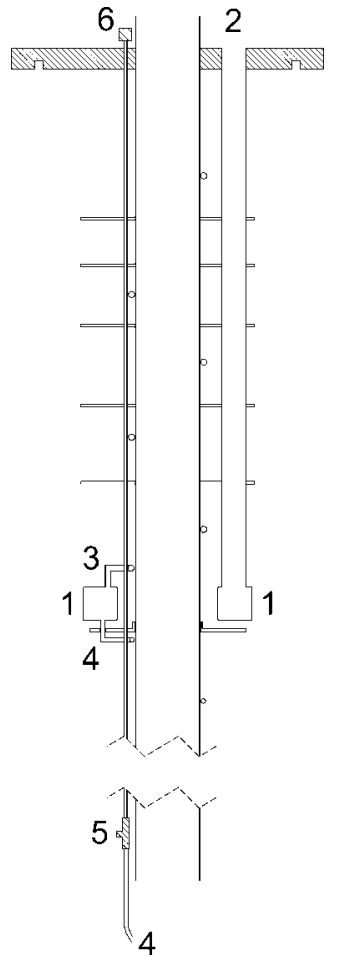

(b)
Figure 7 Schematic drawings of the cryostat. (a) Vacuum mantle, with two heat shields in the vacuum space. The upper, "neck," section sits on top of the outer vacuum case of the magnet. (b) The central access tube with the separator (1), its input port (2) and flow-control capillary (3), and the main capillary (4) with its needle valve (5) controlled from the top flange (6). The flow-control capillary is coiled around the central access tube in the neck section. Its feedthrough through the top flange is not shown. The main capillary is coiled around the tail section of that tube, except the short straight part after the needle valve.

mantle with an indium seal, to maintain vacuum integrity also during initial cool-down. The two heat shields are cooled by thermally anchoring them around the main $\mathrm{He}$ vessel at appropriate levels in the neck of the cryostat.

The cryostat is of the continuous flow type, in which liquid helium is drawn continuously from a non-magnetic storage dewar close to the polarizer magnet through a rigid, inverted- $U$ transfer line into a doughnut-shaped volume, called the separator. The length of the transfer line is kept minimal. In the separator, the splash vapor content, caused by thermal losses in the transfer line, is eliminated via a "flow control capillary" by applying a slight under-pressure with a small membrane pump, operated in a flow controlled mode. This capillary is coiled around the central access tube (which passes through the central hole of the separator) and on the baffles, which furthermore are cooled by the gas boiling off from the main He reservoir. Thus an efficient use of the enthalpy of both gas streams is realized, which results in a low overall consumption.

Liquid helium is supplied to the main volume from the lower part of the separator via the "main capillary" that ends in a variable impedance (a needle valve operated from the top flange). Mechanically, these elements are fitted to the central access tube, that descends from the top flange into the main volume. The upper end of that tube provides access for the different inserts (see "Cryostat Inserts" section) and is equipped with a T-shaped pumping port with two NW50 flanges for a double pumping line (not shown on Fig. 7).

The initial cool-down is carried out using the main pumping system throttled to a constant under-pressure via a mechanical manostat (Leybold MR 16). The pumping system consists of a $250 \mathrm{~m}^{3} / \mathrm{h}$ Roots pump, backed by a rotary vane pump (Leybold Trivac D65B). It could be upgraded to $1,000 \mathrm{~m}^{3} / \mathrm{h}$ for even lower sample temperature.

The level of liquid $\mathrm{He}$ in the main volume is measured by a standard superconducting level meter (Twickenham Scientific Instruments). A small turbo-pumping system provides the isolation vacuum of the cryostat. The stainless-steel pumping lines are electrically insulated from the cryostat by short pieces of rigid plastic tubing with appropriate flanges.

Mainly for diagnostic purposes, silicon diode thermometers (LakeShore DT-470) are mounted on both radiation screens. Two RuO thermometers (LakeShore RX-102A) are placed on the separator and at the outlet of the main capillary into the sample volume; the latter is used as indicator for the bath temperature. We have roughly checked the reading on the sample space thermometer by measuring the $T_{1}$ of very pure $\mathrm{Al}$ foil $\left(T_{1} T=1.80 \pm 0.05 \mathrm{~s} \mathrm{~K}\right)(13)$, but it remains difficult to ascertain the sample temperature with the microwaves on. These thermometers serve to check repeatability from experiment to experiment, rather than to measure the absolute temperature.

In summary, the cooldown sequence is:

1. Use the turbo pump to obtain an initial vacuum of better than $5 \times 10^{-5}$ mbar, as read on the pump input. 
2. Start drawing ${ }^{4} \mathrm{He}$ from the storage dewar into the separator with the membrane pump.

3. With the needle valve fully open, use the rotary pump to draw gas/liquid into the main volume, where the pressure is stabilized by the manostat at 800 mbar. When the level monitor indicates the presence of liquid, adjust the needle valve.

4. Start pumping on the main volume with the full main pumping system.

5. Adjust the needle valve and the gas flow through the membrane pump to obtain a constant temperature at constant ${ }^{4} \mathrm{He}$ level in the main volume.
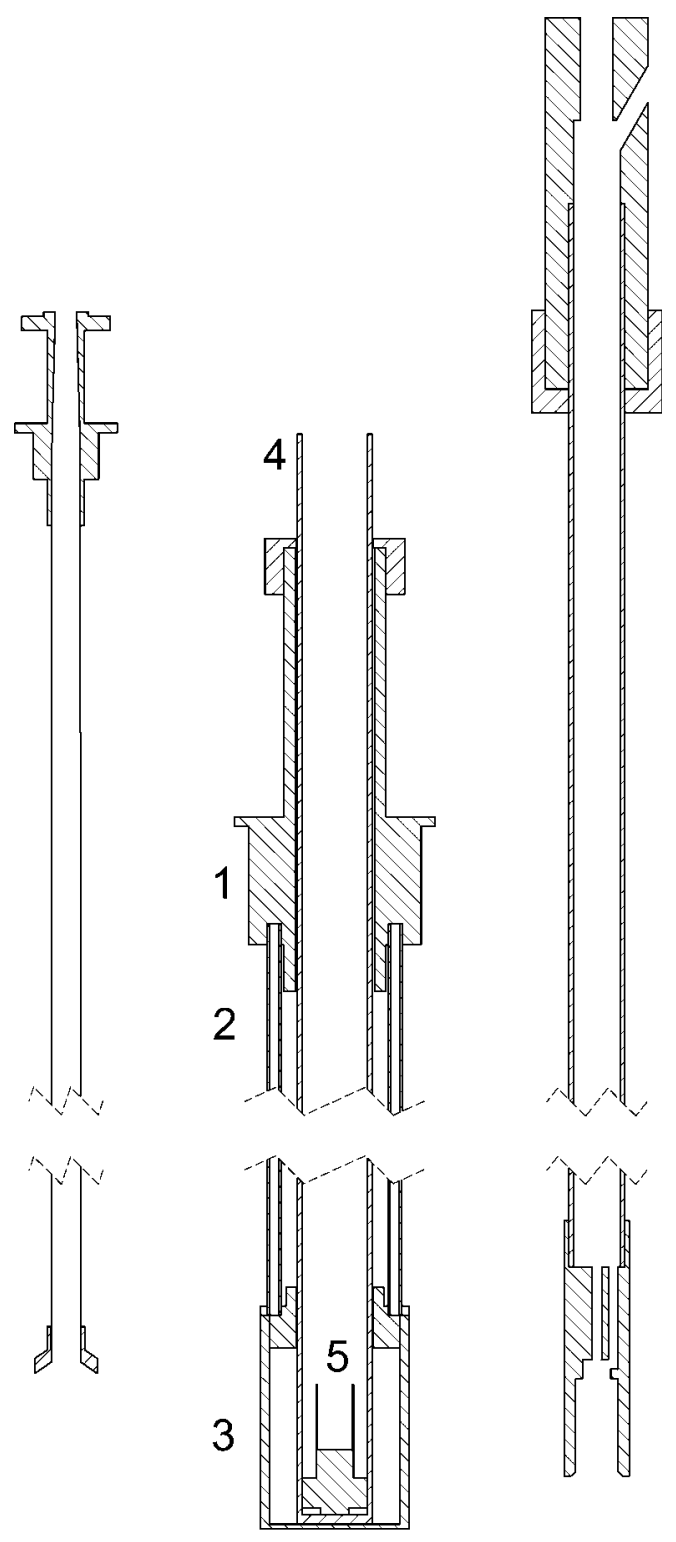

(b) (c)
The last step can be omitted for a simple "oneshot" operation. Under usual circumstances, the amount of ${ }^{4} \mathrm{He}$ in the main cryostat volume is sufficient to polarize a sample, with the membrane pump switched off. But sample throughput is increased when the ${ }^{4} \mathrm{He}$ level is maintained.

\section{Cryostat Inserts}

The set of inserts that we have designed, see Fig. 8, is somewhat different from that proposed in (6). The main consideration is that the elements with a large heat capacity, those that define the mechanical structure, stay always in place at low temperature, while the different functions can be performed by exchanging parts that are mechanically and cryogenically rather simple. Another is that our low-temperature space has a smaller diameter $(37 \mathrm{~mm})$ than that in a special-purpose magnet without room temperature bore tubes (6).

There is an interesting, space-saving difference in how we couple the microwaves to the sample. The design in (6) couples a rectangular WR28 waveguide to the microwave cavity, just as one would do with a monomode ESR cavity. But actually, the DNP experiment is much simpler than ESR, since it only requires that we saturate the ESR transitions everywhere in the sample. The useful sample volume in a monomode ESR cavity is smaller than the 3rd power of half a wavelength, something like 3 microliters at $94 \mathrm{GHz}$, and the total cavity volume is a small multiple of that. A DNP cavity can be much larger.

Formally, the stationary electromagnetic field inside any dielectric or metallic enclosure can always

Figure 8 Schematic drawings of the cryostat inserts. (a) The main insert $(1,2,3)$ with the sample holder insert (4), and sample holder (5) in place. To see just the main insert, imagine sliding upwards the tube (4), including the sample holder (5). The main insert top flange (1) fits into the central access tube of Fig. 7(b) and through thin tubes (2) retains the microwave cavity (3). The NMR coil (not drawn) sits inside the cavity, outside the sample holder insert. (b) The microwave insert. Its main part is a circular tube, its top part a transition from rectangular to circular waveguide, and its bottom part a small cylindrical microwave horn. (c) The dissolution and blow-over insert, very schematically. The capillary tubes are not shown, but the inlet and outlet ports on the top are indicated, as well as the (central) incoming and (sideways) outgoing paths in the bottom part. The bottom part grips firmly around the sample holder (5). The water boiler and its valves, not drawn, sit atop. 
be decomposed into a superposition of eigenmodes, but in our case the eigenmodes are closely spaced compared with their quality factors, so that in practice the enclosure is non-resonant (i.e., has an extremely low overall quality factor). This is all the more true when the enclosure contains a sample, sample holder, NMR coil and other dissipative elements. In that case, the best we can do is to direct the microwaves from the incoming waveguide as directly as possible onto the sample. The idea is more to have at the waveguide end a "horn antenna" that irradiates the sample, while the enclosure simply confines the microwaves. Therefore our coupling structure is geometrically and mechanically much simpler. It is perhaps not quite appropriate to refer to the enclosure as a "(non-resonant) cavity," but this is the commonly used expression in target research.

Main Insert and Sample Holder Insert. The main insert consists of the microwave cavity, inner diameter $28 \mathrm{~mm}$, with the NMR coil inside. It hangs from a number of thin-walled stainless-steel tubes, spaced on a diameter of $26 \mathrm{~mm}$. The top of the cavity has a circular opening of $16.6 \mathrm{~mm}$ diameter, through which enters the sample holder insert (see later). Apart from servicing periods, the main insert remains always in place.

The sample holder insert is a simple glassfiber-epoxy tube closed at the bottom. Near the bottom are two radial windows, in front of the saddle-type NMR coils. Their position is chosen to minimize pickup of unwanted NMR signals, and one of the windows is big enough to put the sample holder in place. The sample holder itself has a useful volume $14 \mathrm{~mm}$ height, and $7.5 \mathrm{~mm}$ diameter. Estimating the packing fraction for the sample beads as 0.7 , the useful bead volume is $0.4 \mathrm{ml}$. During microwave irradiation, this insert is pushed down to bring the sample in the center of the microwave cavity, and below the ${ }^{4} \mathrm{He}$ level. For the dissolution step, it is pulled up above the ${ }^{4} \mathrm{He}$ level (the main insert staying where it is).

Even when the filling factor of the NMR coil is low, because of space requirements for the sample holder insert, its RF efficiency can be optimized by a suitable choice of the circuitry. It is well known from usual low-temperature NMR (14-16) that a circuit with the coil "below" and the tuning capacitor "on top" of a cryostat suffers from excessive losses, that can be interpreted as arising from multiple reflections between coil and capacitor, thereby multiplying the effective length of the, inevitably lossy, connection between the two. While it is often impractical to have variable tuning and matching capacitors "below," the situation can be greatly improved by having a rough tuning and matching, using small-size fixed capacitors, close to the coil, and doing the fine tuning on top, at room temperature (17). This is the scheme we used. It is inherently narrow-banded, because the fixed capacitors determine the tuning. For the observation of ${ }^{13} \mathrm{C}$, we use a saddle coil with two times four turns, $24 \mathrm{~mm}$ height, and $18 \mathrm{~mm}$ diameter. With $\sim 200 \mathrm{~W}$ transmitter power, the length of the fivedegree tipping pulses used to measure the polarization curves is $500 \mathrm{~ns}$. Connections are made through stainless steel coaxial cables (Rosenberger MicroCoax UT-141-SS).

Microwave Insert. The microwave generator output is a rectangular WR10 waveguide. It is protected by a three-port circulator (Elva-1, model CR-10/94/ 2 ), followed by an $E$-plane 90 -degree bend to the vertical. At this point therefore, we expect a pure rectangular $\mathrm{TE}_{10}$ mode. Next comes a tapered rectangular to cylindrical transition, and the mode at its output is expected to be cylindrical $\mathrm{TE}_{11}$. The stainless-steel cylindrical waveguide has an inner diameter of 6.05 $\mathrm{mm}$. Following a practice common in ESR, its lower end is filled with Teflon (not shown in Fig. 8), to eliminate variable reflections when the liquid He surface moves up and down. The precaution is probably not essential for a simple DNP experiment. At the bottom is a conical transition to $13.4 \mathrm{~mm}$ diameter, which forms the "horn antenna" inside the microwave enclosure. No doubt that we no longer excite a pure mode, and the actual situation is much too complicated for analysis. But judging from our experimental results, this simple scheme works quite well. We do not attempt to control the vertical position of the horn with respect to the microwave cavity. Typically, we find the reflected power at the circulator to be better than $-20 \mathrm{~dB}$.

Dissolution and Blow-Over Insert. Once a satisfactory degree of DNP has been reached, as measured by the NMR spectrometer (FID after five-degree tipping pulses), the microwave generator and the pumps are switched off, and the main cryostat volume is brought at a small over-pressure using He gas from a bottle. Next the microwave insert is removed. Spin-lattice relaxation times are long with respect to the time needed for this and the following manual operations. The sample holder insert is pulled above the ${ }^{4} \mathrm{He}$ level, and the dissolution/blow-over insert pushed down inside the sample holder insert tube. Its principle is quite similar to that described in (6). The bottom end of its carbon-fiber main support tube is a solid Teflon piece that fits snugly around the sample 
holder, and from which protract two hollow stainless-steel needles. The needles are connected through Teflon capillaries to the inlet and outlet ports on top of this insert. The inlet port is connected to a $7 \mathrm{ml}$ stainless steel water boiler (in a typical experiment, it is filled with $5 \mathrm{ml}$ water). The boiler temperature is stabilized at a desired temperature, typically $440 \mathrm{~K}$. A set of computer controlled pneumatically-activated valves pressurizes the water with He gas from a cylinder, opens the valve for the boiling water to go down along one capillary tube, and provides a short push of $\mathrm{He}$ gas to propel the so created solution through the other capillary tube towards the separator/infusion pump inside the scanner.

\section{Separator/Infusion Pump}

During the blow-over [see Fig. 9(a)], the piston of the pump is maintained in its "up" position, and the mixture of He gas and liquid sample is blown into the cylinder volume through an entry port just below the piston. The cylinder volume is kept at atmospheric pressure through a similarly placed vent port, via an overflow/reflow volume (in fact, there are three of these). Therefore the gas escapes to the atmosphere, whereas the liquid, possibly after a short stay in the overflow volume, will settle at the bottom

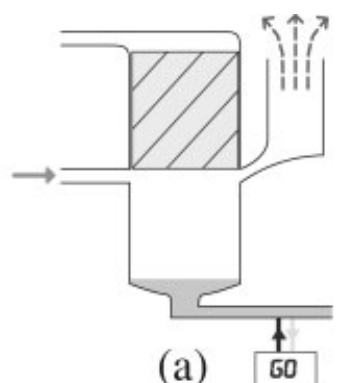

(a)

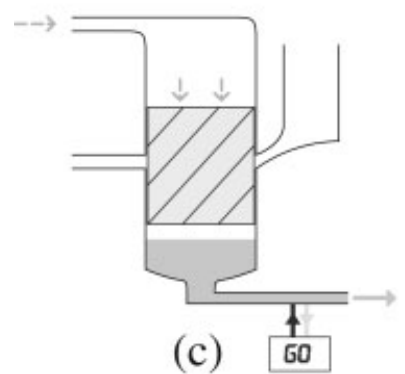

(c)

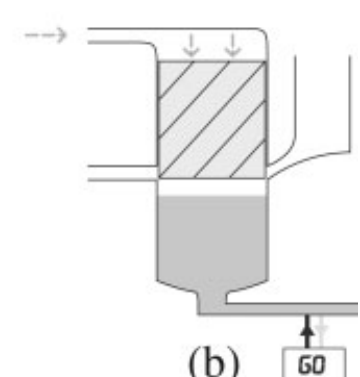

(b)

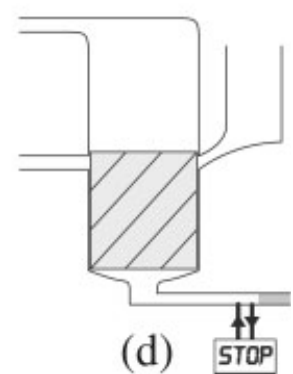

Figure 9 Operation of the separator/infusion pump. The prepolarized solution and its driving $\mathrm{He}$ gas arrive at center left of the device. The gas vents top right. The pump piston (hatched) is driven from top left. The connection to the animal catheter is bottom right. The "go/stop" sign represents the optical bubble detector. of the cylinder volume. Typically, the settling delay is chosen as $1 \mathrm{~s}$. We expect that after this delay the sample is homogenized. Next [Fig. 9(b)], the piston is forced downwards by applying hydrostatic pressure on its other face with a computer controlled pump. The pressure transmitting medium is physiological saline, so, should a small leak along the piston wall exist, this has no serious effect. Initially, the He gas is forced out through the overflow vents, but next the entry and vent ports are closed off by the piston wall. At that point the infusion stage begins [Fig. 9(c)]. The infusion port is located at the bottom of the cylinder, and during animal preparation care has been taken that the infusion catheter and a small volume of the cylinder have been filled with saline, without gas bubbles [Fig. 9(a)].

When the piston stroke comes near its bottom end, a risk exists that trapped He gas might enter the infusion catheter. Therefore the output port is equipped with a fiber-optic bubble detector, that stops the infusion in that case [Fig. 9(d)]. Otherwise, the infusion goes on until a preset volume of pressure-transmitting fluid has been displaced. In most cases we infuse the full available sample volume. It is generally believed that the amount of TEMPO involved does not lead to significant physiological side effects in the animal [see e.g., (18) and references therein].

\section{PERFORMANCE}

\section{Cryogenic Consumption}

The cool-down sequence to $1.1 \mathrm{~K}$ takes between 3 and $4 \mathrm{~h}$ and requires less than $5 \mathrm{l}$ of liquid He. During a typical experiment, including sample loading, He refilling, microwave irradiation, and dissolution, corresponding to an overall time period of $1-2 \mathrm{~h}$, the liquid He consumption is about 2.51 . The amount of liquid He needed for a typical week of experimenting $(\sim 2$ dissolutions per day) is of the order of 601 . The design of the cryostat is such that the storage dewar can be replaced without interrupting the low-temperature (1.1 $\mathrm{K}$ ) experiment in progress. Therefore, if needed, very long low-temperature experiments can be performed even with low-capacity storage dewars. The temperature regulation has good long-term stability, with a maximum variation of $50 \mathrm{mK}$ over more than $12 \mathrm{~h}$.

\section{Solid State Polarization}

Here we describe ${ }^{13} \mathrm{C}$ polarization experiments on three small molecules, acetate, glycine and urea, in glass-forming solvents and with TEMPO as radical. All three molecules are singly labeled in ${ }^{13} \mathrm{C}$. The 


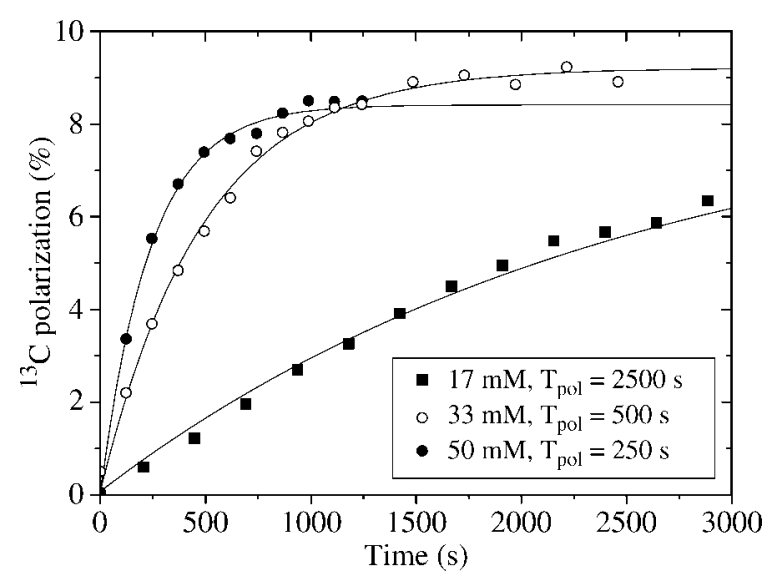

Figure 10 Polarization curves for $3 \mathrm{M}$ acetate in a deuterated solvent, 67/33 water/ethanol, with different concentrations of radical.

solvents were water/ethanol in a $67 / 33$ volume ratio for the acetate, 40/60 water/glycerol for the glycine and pure glycerol for the urea. Only the urea solvent was not deuterated.

Standard operation is at an estimated sample temperature of $1.2 \mathrm{~K}$ (bath temperature at $1.1 \mathrm{~K}$ ), and with $30 \mathrm{~mW}$ of microwave power. For each type of sample, we have checked the range of $10-200 \mathrm{~mW}$, and always find that the polarization hardly increases beyond $20 \mathrm{~mW}$. Each individual polarization curve has been calibrated by the corresponding thermal equilibrium signal at $1.2 \mathrm{~K}$. The uncertainty in the actual sample temperature results in a $10 \%$ uncertainty in the absolute polarization values.

For all three molecules we find that increasing the TEMPO concentration decreases the polarization time constant, but hardly affects the final polarization, as shown in Figs. 10-12. The timescale of Fig.

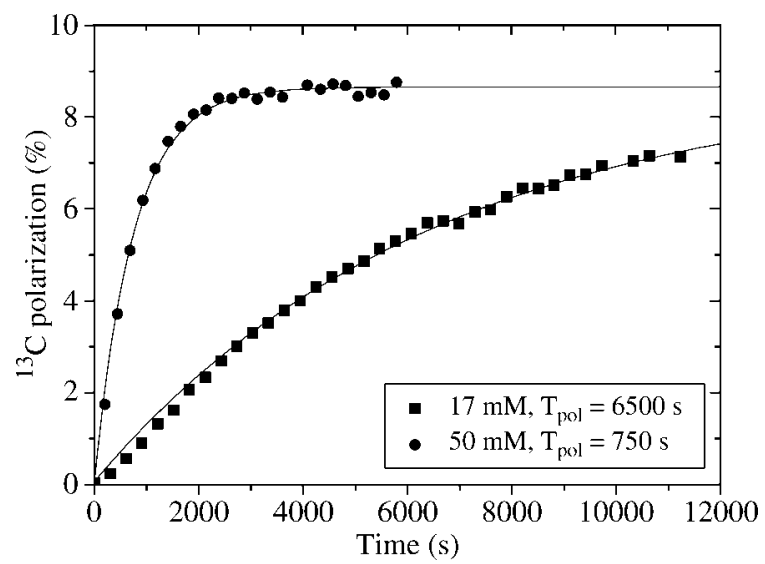

Figure 11 Polarization curves for $1.5 \mathrm{M}$ glycine in a deuterated solvent, 40/60 water/glycerol, with different concentrations of radical.

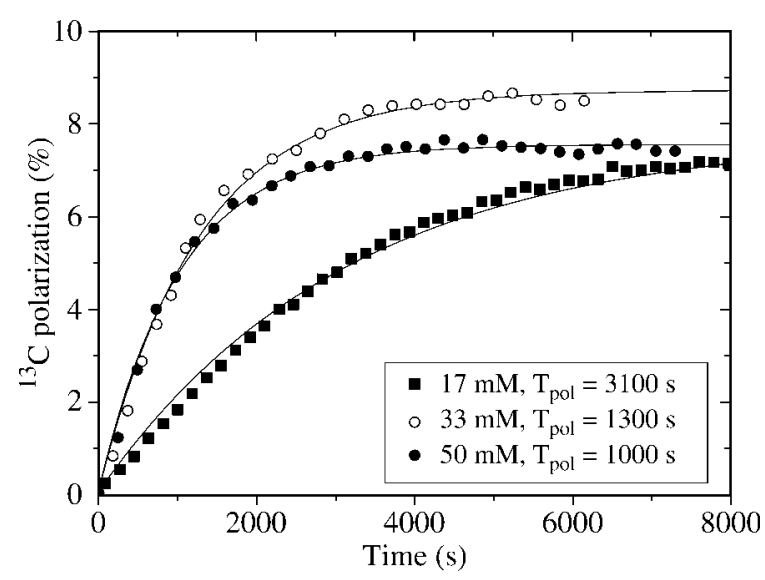

Figure 12 Polarization curves for $8 \mathrm{M}$ urea in undeuterated glycerol with different concentrations of radical.

12 is comparable to that for highest concentrations of radical in Fig. 3 of (7). At constant radical concentration, the polarization times in the three systems are markedly different.

An effect not reported previously, we find that the polarization rate is faster at higher acetate concentrations, see Fig. 13. The final polarizations, however, do not change significantly (not shown). We have observed a similar, although less important, effect for the urea system; in the accessible solubility range of glycine, which is much smaller than the other two, the effect is undetectable (data not shown).

\section{Blow-Over Efficiency}

Finally we present an example that uses the full dissolution/blow-over setup. The sample is glycine with

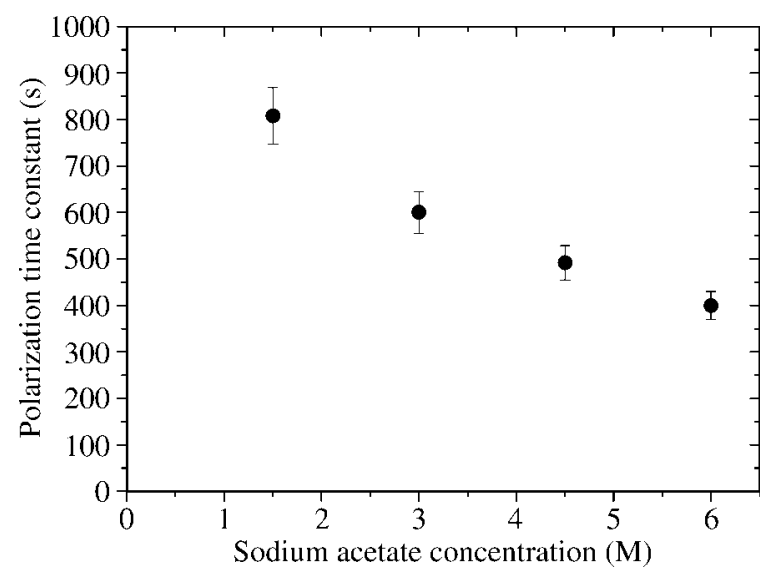

Figure 13 Polarization time constants for acetate with different concentrations of acetate in a deuterated solvent, $67 / 33$ water/ethanol, at a constant radical concentration of $33 \mathrm{mM}$. 


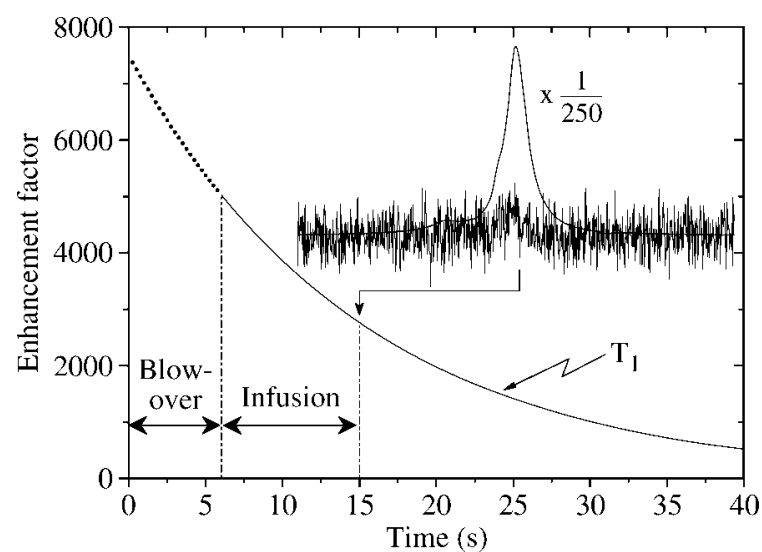

Figure 14 Measured enhancement of the ${ }^{13} \mathrm{C}$ signal amplitude, with respect to its thermal equilibrium value in the 9.4 T MRI field, for labeled glycine in deuterated water/glycerol, after dissolution in $\mathrm{D}_{2} \mathrm{O}$ and blow-over into the MRI magnet. The insert shows the thermal equilibrium signal, and the (scaled) signal immediately after the (simulated) infusion. The sample was collected from the separator/infusion pump, exactly as it would have been for an in vivo experiment, but here a cylindrical phantom tube was used with an adapted RF coil.

$50 \mathrm{mM}$ of radical, for which the polarization curve is shown in Fig. 11. In the MRI magnet, a phantom RF circuit was used, with a solenoidal coil wrapped around a cylindrical tube, into which the separator/ infusion pump delivered the solution, but the timings were exactly what we use for in vivo experiments. The result is shown in Fig. 14. The actual NMR measurement starts $15 \mathrm{~s}$ after the dissolution. We then measure a $T_{1}$ of $15 \mathrm{~s}$. Since all of the solution has arrived in the pump, which is in the MRI field, $9 \mathrm{~s}$ earlier, we can confidentially extrapolate back to that point in time. The extrapolation to zero time is less certain, because the relaxation time during the blowover is not known. Anyway, this extrapolation is about $20 \%$ lower than what would be expected from the polarization in the $3.35 \mathrm{~T}$ prepolarizer magnet, prior to dissolution, Fig. 11. Although we hope to improve on this in the future, it shows that indeed most of the solid-state polarization is retained in the liquid state, as was first demonstrated in $(6,7)$.

\section{DISCUSSION}

The earliest ${ }^{13} \mathrm{C}$ DNP experiment that we are aware of was performed 35 years ago (2). Its main purpose was the identification of the DNP mechanism. Similar ${ }^{13} \mathrm{C}$ DNP experiments have been reported in (1921). The DNP-MAS experiments $(3,4)$ did not involve very low temperatures, and will not be further discussed here. DNP of ${ }^{2} \mathrm{H}$ has been more frequent, since it can both test theory and create useful targets. Data in $(19,20)$ are particularly interesting, since they involve all three nuclei. An early discussion of ${ }^{1} \mathrm{H}$ and ${ }^{2} \mathrm{H}$ data related to the theory of thermal mixing is (22); the most complete still is (20). A summary of literature data on DNP in glassy samples is given in Table 1. All numerical values have been scaled to our experimental conditions, $T=1.2 \mathrm{~K}$ and $B_{0}=3.35 \mathrm{~T}$. ESR parameters for the usual paramagnetic centers in deuterated butanol at $2.5 \mathrm{~T}$ and $1 \mathrm{~K}$ have been published recently (26), and most values in our table are taken from that paper, assuming that both the ESR line width and the $g$-anisotropy, expressed in frequency units, scale linearly with field. The numerical values given in Table 1 for the widths of the microwave spectra have been scaled from the

Table 1 DNP in Glassy Samples

\begin{tabular}{|c|c|c|c|c|c|c|c|c|c|}
\hline Radical & $\mathrm{FW}$ & $\Delta g$ & Extr & Solvent & Nuclei & $B$ & $T$ & $T_{\mathrm{s}}$ & Ref \\
\hline \multirow[t]{5}{*}{ Porphyrexide } & 196 & 377 & 470 & ${ }^{13} \mathrm{C}$-Butanol & ${ }^{1} \mathrm{H},{ }^{13} \mathrm{C}$ & 2.527 & 0.48 & 5.4 & 2 \\
\hline & & & 470 & Butanol & ${ }^{1} \mathrm{H}$ & 2.5 & 1.05 & 5.1 & 23 \\
\hline & & & & Ethanol/water & ${ }^{1} \mathrm{H}$ & 2.5 & 1.05 & 6.4 & 1 \\
\hline & & & & Butanol & ${ }^{1} \mathrm{H}$ & 3.5 & 0.5 & 8.2 & 1 \\
\hline & & & 435 & Butanol & ${ }^{1} \mathrm{H}$ & 5 & 0.96 & 8.7 & 24 \\
\hline Cr-V Dichromate & & 380 & 440 & $\left(\mathrm{CD}_{2} \mathrm{OH}\right)_{2}$ & ${ }^{1} \mathrm{H},{ }^{2} \mathrm{H},{ }^{13} \mathrm{C}$ & 2.5 & 0.38 & 2.9 & 20 \\
\hline Cr-V EHBA & 462 & 562 & 530 & $\left({ }^{13} \mathrm{CH}_{2} \mathrm{OH}\right)_{2}$ & ${ }^{1} \mathrm{H},{ }^{13} \mathrm{C}$ & 2.527 & 0.4 & 3.6 & 21 \\
\hline BDPA & 20 & 130 & 147 & D-Toluene & ${ }^{2} \mathrm{H},{ }^{13} \mathrm{C}$ & 2.5 & 0.7 & 6.4 & 19 \\
\hline TAM & 32 & 26 & 74 & D-Propanediol & ${ }^{2} \mathrm{H}$ & 2.5 & 1 & 3.6 & 25 \\
\hline TAM & 44 & & 85 & $\left(\mathrm{C}_{3} \mathrm{D}_{4}\right)\left(\mathrm{CD}_{2} \mathrm{OH}\right)_{2}$ & ${ }^{13} \mathrm{C}$ & 3.35 & 1.1 & 3.4 & 7 \\
\hline
\end{tabular}

The ESR FWHM line width "FW" and anisotropy $\Delta g$ are in MHz. Under "Extr" is the distance between the extrema of the microwave spectrum, in MHz. $B$ and $T$ give the field (in T) and bath temperature (in $\mathrm{K}$ ) of the original experiment. $T_{\mathrm{s}}$ is the scaled spin temperature in $\mathrm{mK}$, expected at $3.35 \mathrm{~T}$ and $1.2 \mathrm{~K}$ for the specified nuclei in the given solvent. 
original data linearly with field and should be considered as an illustrative estimate only. At $3.35 \mathrm{~T}$, twice the Larmor frequency is $285 \mathrm{MHz}$ for ${ }^{1} \mathrm{H}, 44 \mathrm{MHz}$ for ${ }^{2} \mathrm{H}$, and $72 \mathrm{MHz}$ for ${ }^{13} \mathrm{C}$. The spin temperatures $T_{\mathrm{s}}$ have been scaled with $B / T$, according to the model of "Borghini Model" section.

\section{Radicals}

A number of different radicals appears in the list, some of which are no longer in frequent use. For a recent review of radical chemistry, see e.g., (27).

Porphyrexide is a nitroxide radical that has been known for more than a century. It is no longer commercially available, and in target work it has been largely replaced with TEMPO. It has mainly been used in alcohols (or alcohol/water mixtures), particularly butanol. The best proton results in ethanol/water mixtures, see Fig. 5 of $(1)$, correspond to ${ }^{1} T_{\mathrm{s}}(2.5 \mathrm{~T}$, $1.05 \mathrm{~K})=7.5 \mathrm{mK}$. Those data indicate very low polarizations $(\approx 1 \%)$ for pure water. Part of the reason may be that pure water samples hardly can be obtained as well-formed glasses; perhaps solubility of the radical is another.

The nitroxide radical TEMPO is probably the most widely used wherever stable radicals are needed. It can be obtained commercially from several sources. The ESR parameters are very close to those of porphyrexide (26). Most of the target work using TEMPO has been performed at very low temperature, see e.g., (28), and is therefore of little use for comparison with our standard values of $3.35 \mathrm{~T}$ and 1.2 K. However, the maximum deuteron polarization in deuterated butanol at these low temperatures is very similar for TEMPO and porphyrexide, see e.g., Table 1 of (26).

The $\mathrm{Cr}^{\mathrm{V}}$ complexes are known to be particularly efficient for the polarization of diols. They come in two varieties: the paramagnetic centers that are prepared in situ from potassium dichromate in diols, and the EHBA or EDBA (the deuterated form) complexes that are available as powders. The ESR data for EDBA in butanol have been given in (26). For the in situ formed complex, a value $\Delta g / g \approx 4 \times 10^{-3}$ has been estimated $(8)$.

BDPA, bisdiphenylene-phenylallyl, does not dissolve readily in alcohols, and is not included in the ESR data of (26). Its ESR parameters in Table 1 have been estimated from Fig. 8 of (8).

Members of the TAM radical family have been known for a long time, but only recently certain proprietary variants [see the literature quoted in (29)] have been shown of great use in DNP experiments, in particular for low- $\gamma$ nuclei $(6,7,25)$. The formula
$\left(\mathrm{C}_{3} \mathrm{D}_{4}\right)\left(\mathrm{CD}_{2} \mathrm{OH}\right)_{2}$ in Table 1 refers to a substituted cyclopropane.

\section{Pure Solvents}

Only results on pure solvents have been included in Table 1, the results on solute molecules will be discussed in the next sections. According to their ESR characteristics, we can distinguish the "broad line" group of nitroxide radicals and $\mathrm{Cr}^{\mathrm{V}}$ centers from the "narrow line" group BDPA and TAM. The table gives no ${ }^{1} \mathrm{H}$ data for the narrow-line radicals, because here protons are mainly polarized through the solid effect, as can be understood from comparing the ESR line width parameters with twice the proton frequency $(285 \mathrm{MHz})$. Examples of proton polarization (by the solid effect) can be found in (8) for BDPA and in (7) for TAM. From the equality of ${ }^{1} \mathrm{H}$ and ${ }^{13} \mathrm{C}$ spin temperatures for the broad line group, we conclude that with these radicals both nuclei are polarized through thermal mixing, which is also indicated by the widths of the microwave spectra.

The lowest spin temperatures are obtained not so much with a given radical, but rather when the solvent is a deuterated diol (although the substituted cyclopropane might be a class apart, see later). Good results on ${ }^{2} \mathrm{H}$ have been obtained both with the broad-line $\mathrm{Cr}^{\mathrm{V}}$ and with narrow-line TAM. The undeuterated ethanediol (21) performs somewhat worse, which could be ascribed to the larger "heat load" effect of protons, as compared with deuterons. For propanediol this effect has been studied in some detail in (8).

The porphyrexide/butanol data show two groups of $T_{\mathrm{s}}$ values, around 5 and around $8 \mathrm{mK}$. The best results are scaled from two experiments that were both performed at $B_{0}=2.5 \mathrm{~T}$, the two others were done at 3.5 and $5 \mathrm{~T}$. It has been noted before, e.g., in Fig. 7 of (8), that broad-line radicals tend to perform below expectations at higher applied fields. One possible explanation is that the line width increases linearly with field, whereas the dipole-dipole coupling is field independent, so that it becomes more and more difficult for the electron spin system to maintain a uniform electron spin temperature across the line (8). For later reference, we note that the ethanol/water result has also been obtained at $2.5 \mathrm{~T}$, so that it may well turn out that an experiment at $3.35 \mathrm{~T}$ would actually yield a spin temperature above the scaled value of $6.4 \mathrm{mK}$. As mentioned before, it is believed that porphyrexide and TEMPO radicals should behave very similarly.

Concerning the polarization mechanism with TAM radicals, the ${ }^{13} \mathrm{C}$ nucleus may be a borderline case for thermal mixing. The extrema in the 
$\left(\mathrm{C}_{3} \mathrm{D}_{4}\right)\left(\mathrm{CD}_{2} \mathrm{OH}\right)_{2}$ microwave spectrum are separated by $85 \mathrm{MHz}$, whereas twice the ${ }^{13} \mathrm{C}$ Larmor frequency in this field equals $72 \mathrm{MHz}$. Furthermore, at these points in the microwave spectrum, no significant cooling of ${ }^{1} \mathrm{H}$ was detected. These characteristics support the idea of a differential solid effect (see Section II), as the original authors have noted (7). The lower gyromagnetic ratio of ${ }^{2} \mathrm{H}$ makes it more likely that this nucleus polarizes through thermal mixing (25); and this will certainly be the only mechanism for ${ }^{15} \mathrm{~N}$.

\section{Dissolved Molecules with TAM}

The biologically most interesting application of the prepolarization technique is to dissolved molecules. There are of course no such examples in the target literature, and so far the number of published papers in the NMR/MRS/MRI field that discuss DNP in the solid state is still rather small.

The first reports on hyperpolarization by DNP ( 6 , 7) mention a sample of ${ }^{13} \mathrm{C}$-labeled urea in pure glycerol as solvent, with $15 \mathrm{mM}$ TAM radical. After some $8 \mathrm{~h}$ of irradiation at nominally $100 \mathrm{~mW}$, the ${ }^{13} \mathrm{C}$ spin temperature was determined as ${ }^{13} T_{\mathrm{s}}(3.35 \mathrm{~T}$, $1.1 \mathrm{~K})=2 \mathrm{mK}$, a value never realized for any nucleus in targets at this bath temperature and field. For a similar sample (20 $\mathrm{mM}$ of radical), the extrema in the ${ }^{13} \mathrm{C}$ microwave spectrum were separated by 63 $\mathrm{MHz}$ (7), again comparable to twice the ${ }^{13} \mathrm{C}$ Larmor frequency. It seems possible that this efficient cooling is directly related to a happy matching of the ${ }^{13} \mathrm{C}$ Larmor frequency and the ESR line width of the radical, and that nor deuterons, nor protons in the sample are cooled as efficiently. Indeed, it is remarkable that the solvent was not deuterated, which usually is disadvantageous for thermal mixing, because of the proton "heat load" (see "Thermal Mixing" and "Pure Solvents" section). But in the case of urea/TAM, the protons of the glycerol cannot load the heat capacity of the electron spins [compare Fig. 2 of (7)] whereas deuteration would have loaded it, at least partially, with the deuterons [compare Fig. 9 of (25)].

It has been found, see Fig. 3 of (7), that there is a very marked dependence of final ${ }^{13} \mathrm{C}$ polarization on the radical concentration, contrary to our results in Figs. 10-12. As mentioned in Section II, such a dependence can become important with the solid effect as polarization mechanism, whereas it should be a minor effect for thermal mixing.

\section{Dissolved Molecules with TEMPO}

Our data for three small ${ }^{13} \mathrm{C}$ labeled molecules as solute in different solvents all show roughly the same final ${ }^{13} \mathrm{C}$ spin temperature, around $10 \mathrm{mK}$, independent of solvent system and concentration of radical or solute. At least for the alcohol-based solvent used for the acetate, deuteration improves the results somewhat (these data will be published elsewhere), as has been seen in similar solvent-only systems, see "Pure Solvents" section. Although final spin temperatures are largely independent of TEMPO and solute concentration, the ${ }^{13} \mathrm{C}$ polarization rate increases with increasing concentrations of either. The polarization rate is thus not simply related to the ratio (solute molecules)/(radical molecules). That the rate increases with increasing radical concentration can be expected on general grounds; the increase with solute concentration suggests that nuclear spin diffusion may play a role.

When comparing our results to those in Table 1 , the first qualification to be made is that the solvents we have tested so far have never been used as targets. For TEMPO-based targets, only pure alcohols have been used. The target results on butanol/water and ethanol/water with porphyrexide show that water is an unfavorable solvent. Our choice of the 67/33 water/ethanol solvent has been suggested by biological considerations, and we have not yet studied the effect of changing the composition. In fact, our final ${ }^{13} T_{\mathrm{s}} \approx 10 \mathrm{mK}$ is much better than would be expected from the extrapolations to high water concentration of the results shown in (1).

So, although the TEMPO radical performs less well than does the TAM radical for urea in glycerol, the result in water-rich solvents seems to be quite adequate, with an improvement in speed of polarization as bonus. On basis of Fig. 4 we suppose that with "broad-line" radicals, it is essential that the sample temperature be kept low. This requires of course in the first place a cryostat with a low bath temperature. The thermometer in our He bath usually indicates values in the $1-1.1 \mathrm{~K}$ range. It is also important to have a well-designed microwave structure, that leads to minimal sample heating.

\section{CONCLUSION}

At least for ${ }^{13} \mathrm{C}$ NMR/MRI/MRS of small molecules, the methods first proposed in $(6,7)$ are clearly promising, even when a rather standard "broad line" radical is used as the paramagnetic center. Our comparison of results, old and recent, in the literature shows that for further progress in this application of DNP, renewed fundamental work on the details of DNP mechanisms certainly will be very useful. 


\section{ACKNOWLEDGMENTS}

Our work is supported by the Swiss National Science Foundation, grant 200021-109479, by the Fund of the Dean of the Faculty of Basic Sciences, EPFL; and by the PSI. We thank the Centre d'Imagerie Biomedicale (CIBM) of the UNIL, UNIGE, EPFL, CHUV, and HUG for access to its facilities. The CIBM is supported by the Jeantet and Leenaards Foundations.

\section{REFERENCES}

1. Mango S. 2004. Early target material research with chemical dopants. Nucl Instr Methods A 526:1-6.

2. Borghini M, Udo F. 1973. Dynamic polarization of ${ }^{13} \mathrm{C}$ nuclei in 1-butanol. Phys Lett A 43:93-94.

3. Wind RA, Duijvestijn MJ, van der Lugt C, Manenschijn A, Vriend J. 1985. Applications of dynamic nuclear polarization in ${ }^{13} \mathrm{C}$ in solids. Progr NMR Spectr 17:33-67.

4. Gerfen GJ, Becerra LR, Hall DA, Griffin RG, Temkin RJ, Dingel DJ. 1995. High frequency (140 GHz) dynamic nuclear polarization: polarization transfer to a solute in frozen aqueous solution. J Chem Phys 102:9494-9497.

5. Farrar CT, Hall DA, Gerfen GJ, Inati SJ, Griffin RG. 2001. Mechanism of dynamic nuclear polarization in high magnetic fields. J Chem Phys 114:4922-4933.

6. Ardenkjaer-Larsen JH, Fridlund B, Gram A, Hansson $\mathrm{G}$, Hansson L, Lerche MH, et al. 2003. Increase in signal-to-noise ratio of $>10000$ times in liquid-state NMR. Proc Natl Acad Sci USA 100:10158-10163.

7. Wolber J, Ellner F, Fridlund B, Gram A, Jóhannesson H, Hansson G, et al. 2004. Generating highly polarized nuclear spins in solution using dynamic nuclear polarization. Nucl Instr Methods A 526:173-181.

8. de Boer W. 1976. Dynamic orientation of nuclei at low temperatures. J Low Temp Phys 22:185-211.

9. Abragam A, Goldman M. 1982. Nuclear Magnetism: Order and Disorder. Oxford: Clarendon Press.

10. Redfield AG. 1955. Nuclear magnetic resonance saturation and rotary saturation in solids. Phys Rev 98: 1787-1809.

11. Solomon I. 1962. Polarisation dynamique et détection des signaux de résonance par double irradiation. Proc Coll Amp 11:25-38.

12. Borghini M. 1968. Spin-temperature model of nuclear dynamic polarization using free radicals. Phys Rev Lett 20:419-421.

13. Anderson AG, Redfield AG. 1959. Nuclear spinlattice relaxation in metals. Phys Rev 116:583-591.
14. MacLaughlin DE. 1989. Coaxial cable attenuation in NMR sample coil circuits. Rev Sci Instrum 60:32423248.

15. Walton JH, Conradi MS. 1989. Probe tuning adjustments-need they be in the probe? J Magn Reson 81:623-627.

16. Kuhns PL, Lee SH, Coretsopoulos C, Hammel PC, Gonen O, Waugh JS. 1991. A low-temperature NMR probe for use in a dilution refrigerator. Rev Sci Instr 62:2159-2162.

17. Conradi MS. 1993. Low-temperature NMR techniques. Concepts Magn Reson 5:243-262.

18. Soule BP, Hyodo F, Matsumoto K, Simone NL, Cook JA, Krishna MC, et al. 2007. The chemistry and biology of nitroxide compounds. Free Radic Biol Med 42:1632-1650.

19. Borghini M, de Boer W, Morimoto K. 1974. Nuclear dynamic polarization by resolved solid-state effect and thermal mixing with an electron spin-spin reservoir. Phys Lett A 48:244-246.

20. de Boer W, Borghini M, Morimoto K, Udo F. 1974. Dynamic polarization of protons, deuterons and carbon-13 nuclei. J Low Temp Phys 15:249-267.

21. Jarmer JJ, Penttilä S, Hill D, Kasprzyk T, Krumpolc M, Barlett ML, et al. 1986. Dynamic polarization of ${ }^{13} \mathrm{C}$ in ethylene-1,2- ${ }^{13} \mathrm{C}_{2}$ glucol. Nucl Instr Methods A 250:576-578.

22. Borghini M, Scheffler K. 1971. Experimental evidence for dynamic nuclear polarization by cooling of electron spin-spin interactions. Phys Rev Lett 26:1362-1365.

23. Mango S, Runólfsson O, Borghini M. 1969. A butanol polarized proton target. Nucl Instr Methods 72:45-50.

24. Hartmann G, Hubert D, Mango S, Morehouse CC, Plog K. 1973. Proton polarization in alcohols at 50 kG, 1K. Nucl Instr Methods 106:9-12.

25. Goertz ST, Harmsen J, Heckmann J, Hess Ch, Meyer W, Radtke E, et al. 2004. Highest polarizations in deuterated compounds. Nucl Instr Methods A 526:33-52.

26. Heckmann J, Meyer W, Radtke E, Reicherz G, Goertz S. 2006. Electron spin resonance and its implication on the maximum nuclear polarization of deuterated solid target materials. Phys Rev B 74:134418.

27. Hicks RG. 2007. What's new in stable radical chemistry? Org Biomol Chem 5:1321-1338.

28. Plückthun M, Bradtke Ch, Dutz H, Gehring R, Goertz St, Harmsen J, et al. 1997. Polarization measurements of TEMPO-doped butanol targets. Nucl Instr Methods A 400:133-136.

29. Reddy TJ, Iwama T, Halpern HJ, Rawal VH. 2007. General synthesis of persistent trityl radicals for EPR imaging of biological systems. J Org Chem 67:46354639. 